\title{
Self-adjustment mechanisms and their application for orthosis design
}

\author{
Viet Anh Dung Cai · Philippe Bidaud • Vincent Hayward • \\ Florian Gosselin
}

Received: 20 December 2015/Accepted: 1 November 2016/Published online: 14 November 2016

(C) Springer Science+Business Media Dordrecht 2016

\begin{abstract}
Medical orthoses aim at guiding anatomical joints along their natural trajectories while preventing pathological movements, especially in case of trauma or injuries. The motions that take place between bone surfaces have complex kinematics. These so-called arthrokinematic motions exhibit axes that move both in translation and rotation. Traditionally, orthoses are carefully adjusted and positioned such that their kinematics approximate the arthrokinematic movements as closely as possible in order to protect the joint. Adjustment procedures are typically
\end{abstract}

Electronic supplementary material The online version of this article (doi:10.1007/s11012-016-0574-0) contains supplementary material, which is available to authorized users.

V. A. D. Cai $(\square)$

Ho Chi Minh city University of Technology and Education, Ho Chi Minh, Vietnam

e-mail: dungcva@hcmute.edu.vn

P. Bidaud

ONERA - Chemin de la Hunire, 92120 Palaiseau, France

e-mail: bidaud@isir.upmc.fr

V. Hayward

Sorbonne Universites, UPMC Univ Paris 06, UMR 7222,

ISIR, 75005 Paris, France

e-mail: hayward@isir.upmc.fr

F. Gosselin

Interactive Robotics Laboratory, CEA, LIST,

91190 Gif-sur-Yvette, France

e-mail: florian.gosselin@cea.fr long and tedious. We suggest in this paper another approach. We propose mechanisms having intrinsic self-aligning properties. They are designed such that their main axis self-adjusts with respect to the joint's physiological axis during motion. When connected to a limb, their movement becomes homokinetic and they have the property of automatically minimizing internal stresses. The study is performed here in the planar case focusing on the most important component of the arthrokinematic motions of a knee joint.

Keywords Self-adjustment $\cdot$ Singular mechanisms . Orthosis design

\section{Introduction}

Medical orthoses serve a number of purposes. One of their function is to compensate for abnormal joint mobility associated with mechanical deficits. They can also contribute to protect a joint from internal injuries due to overstressing or overstretching. Functionally, they must guide the anatomical joint along a desired set of trajectories and prevent undesired movements to take place. These functions can be obtained by means of mechanisms designed to provide mechanical constraints that are reciprocal to the healthy mobility of a joint. At the same time, the free movements of the joint must be such that residual stresses impinging on the limbs be minimized during large joint excursions. 
Compensation for abnormal mobility implies that the combined limb-orthosis structure be overconstrained. The mobility, $m$, of the orthosis, must be smaller than the dimension of the associated space [(e.g. $m<\operatorname{dim}(\mathrm{SE}(3))]$. Movement restriction implies the design of mechanical constraints needed to protect the joint. The protection function of the joint sets requirements on the kinematics of the orthosis in order to fit the joint it protects.

\subsection{General kinematic properties of the human knee joint}

Unlike mechanical joints of ordinary machines, anatomical joints are mobile in all directions. However, the geometry of the articular surfaces in contact, the ligaments, and the muscles acting through the tendons force a joint to move along preferred trajectories according to the load applied, the muscle cocontraction activation, and other factors. As a result, standard lower pairs, or even higher pairs, can only serve as mere gross approximations and some standardization is required to model the joint's motions [1].

Measurements show that, for instance, the instantaneous axis of rotation of even a 'simple' joint such as the knee is far from being fixed. The so-called arthrokinematic motion depends on the bone surfaces geometry, among other factors. The anatomical details of the articular surfaces, the organization of the ligaments, capsules and menisci, combined with the influence of various pathologies owing to age, trauma, or habitual activity determine its kinematics. Complex behaviour partly results from the fact that knee movements involve a combination of rolling and sliding movements of the femur relatively to the tibia combined with the action of the cruciate ligaments [2]. It is nevertheless possible to develop a kinematic model for the knee joint, taking into account ligaments, capsules and the geometry of the articular surfaces [3-5], but the question of parameter identification always remains. To this end, electromechanical devices being able to measure the kinematics, the stability, the rigidity and the laxity of the knee joint in patients suffering knee injuries were designed as described in [6].

Several studies show that the movements of a healthy knee include a principal flexion-extension movement with an amplitude which is conventionaly represented to vary between $0^{\circ}$ and $5^{\circ}$ in extension and between $-130^{\circ}$ and $-150^{\circ}$ in flexion [7]. Since the femoral condyles' surfaces are approximately twice as long as that of the tibial plateau, the knee movement is not simply a rolling movement. During joint flexion, the movement of the femoral condyles is a compound motion comprising three phases described as follows:

1. A rolling phase takes place when the flexion angle is smaller than $-15^{\circ}$ for the internal condyle and up to $-20^{\circ}$ for the external condyle,

2. A rolling and sliding phase occurs when the flexion angle is between $-15^{\circ}$ and $-120^{\circ}$ approximatively,

3. A sliding phase arises from $-120^{\circ}$.

As a consequence, the position of the knee joint axis varies in the range of several millimeters and its orientation fluctuates by several degrees. The displacement of the femoral condyles on the tibial plateau is in the range of $0.8-1.2 \mathrm{~cm} \mathrm{[8]} \mathrm{and} \mathrm{the} \mathrm{angular}$ deviation is of the order of $10^{\circ}$ [9]. It should also be noticed that while the knee is almost blocked at complete extension and that only flexion is possible, when fully flexed, internal rotation becomes possible and the knee lets the tibia rotate around its axis with amplitudes of the order of $\pm 30^{\circ}$. All these movements that occur around the joint axis define the so called arthrokinematics of the joint. Figure 1a, following the methodology proposed in [10] and using the data from [9], gives the visualisation of the variations of the instantaneous joint axis configuration. The curled surface represents the intersection of the moving axis with a family of sagittal planes parametrized by their distance from the axis of the tibia.

\subsection{Usual guidelines for orthosis design}

The design of orthotic mechanisms is often arranged so that the center of rotation of pairs of simple mechanical joints located on each side of the limb move along desired trajectories. An example of such realization is the so-called polycentric joint, which can be seen in several passive orthosis designs. In this design, illustrated in Fig. 1b, the instantaneous center of rotation of one of the articulated side-members is made to depend on the relative position of the two braces [11-14].

However, even with such advanced designs, the effectiveness of a medical orthosis is conditioned by 


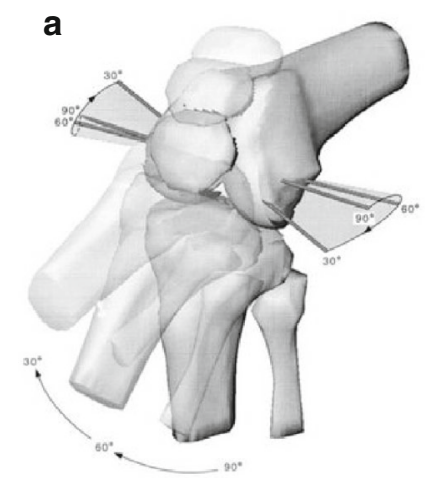

Fig. 1 a Graphical representation of the knee instantaneous helical axis [9] (the variations in the knee axis are amplified for better visibility, the curves generated by the intersection of the knee axis with different sagital planes should be reproduced by

the ability of the orthopedist, or of the user, to affix it on the limb segments so that its axis of rotation remains as close as possible to the anatomical joint it protects. This requires a long and tedious adjustment procedure. One typically accomplishes customization through plastic deformations of the orthosis structure or through modifications of the attachment interfaces [15]. Shortcomings of this approach are that the constraints imposed are always approximative and that attachments can never be rigid. The same principle is applied to both semi-active, or active orthoses which are designed to provide additional torque control to the anatomical joint in order to gradually recover the sensori-motor system. One can cite the knee brace designed with springs to assist stand to sit and sit to stand motion [16], or knee powered orthoses designed to correct the user's movements in the stance phase [17] or to provide rehabilitation exercise for patients after stroke [18].

\subsection{The self-adjusting mechanism approach}

We present in what follows a novel approach to the problem of meeting an orthosis functional requirements that do not involve manual intervention and illustrate it in the case of the human knee. Additional degrees of freedom (DOFs) that permit the complete mechanism (the anatomical joint plus the external orthosis) to reconfigure itself so that the orthosis axis self-aligns optimally with the varying instantaneous joint axis are introduced. This paper extends the

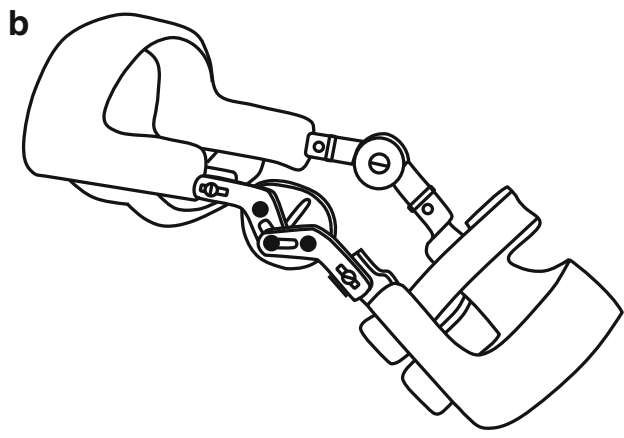

the instantaneous center of rotation of medical orthoses). b Example of knee orthosis with one polycentric joint-Image adapted from Herzberg et al. [12]

results of previous research [19] by presenting a more complete model of the horizontal self-adjustment movement together with the analysis of the vertical self-adjustment movement. In contrast to other designs, employed more particularly in active orthoses, where additional DOFs remain free all throughout the joint movements [20-25], we focus in this work on passive orthoses. The extra DOFs are blocked after a short period of self-adjustment, after which the orthosis can work normally to protect the anatomical joint.

For the sake of exposition, the analysis performed in this paper assumes that the knee joint can be modeled as a simple pivot joint (i.e. a revolute joint$\mathrm{R})$. Indeed, one can note from Sect. 1.1 that the knee flexion is of much greater amplitude than the other components of the complex knee arthrokinematic motion. Hence, an orthosis able to naturally follow this motion, even approximatively, should already greatly reduce potential stresses of the knee due to a wrong installation of the orthosis on the leg. Also, focusing on simpler planar mechanisms allows one to simplify the calculations and to better analyze the behavior of the linkage during different operating phases. Further studies will be conducted for spatial knee models in the future.

Despite the fact that real anatomical joints are compliant owing to the presence of visco-elastic tissues, we will consider a knee joint to be a rigid pivot assuming that the parasitic movements permitted by the knee compliance are small compared to the knee flexion. It would nevertheless be interesting to 
take the knee joint compliance into account in future studies [26].

The additional DOFs can be prismatic joints (P), introduced on both sides of the orthosis main joint, such that, when considering the most simple systems using a simple revolute joint $(\mathrm{R})$ to guide the knee movement, the enhanced orthosis becomes a PRP mechanism. The same principle would apply to polycentric (PC) joint orthoses, which are usually designed to follow the complex movements of a real knee joint. In this case we would employ a P-PC-P mechanism to enhance this type of orthoses and include the selfadjustment property.

We consider two cases. In the first, we study what we term the horizontal self-adjusting mechanisms, where the orthosis is free to move in the direction of the thigh and of the calf such that, after selfadjustment, the orthosis axis is in their bisecting plane. In the second case, we consider what we term the vertical self-adjusting mechanisms. The latter mechanism allows the orthosis to move in a direction perpendicular to the thigh and to the calf. By combining these two mechanisms, two self-adjustment movements can be performed consecutively, as will be further explained in Sect. 2.2, ensuring that the orthosis and the knee axis become aligned.

This article is organized as follows. These two types of mechanisms are first introduced in Sect. 2. Then horizontal and vertical self-adjustment movements are more specifically studied and analyzed in Sects. 3 and 4. In Sect. 5, horizontal and vertical selfadjusting mechanisms (both of PRP type) are simulated in order to validate the theoretical analysis. Finally, Sect. 6 discusses the results and concludes.

\section{Self-adjustment property}

\subsection{Introduction of two basic types of self- adjusting mechanisms}

The mechanisms under study are shown together with the knee joint in Figs. 3 and 4, which for the purpose of simplicity, is represented here by a hinge joint. These two mechanisms include a main hinge joint (R) plus two additional prismatic joints $(\mathrm{P})$. Auxiliary rotational joints, at $D_{1}$ and $D_{2}$, are included to represent the torsional elasticity of the attachments, as further described below. The resulting mechanisms are of type PRP. When connected with the knee joint they form a closed-chain mechanism which has only one degree of freedom. However, when the two prismatic joints are aligned, i.e. when the mechanisms are in a singular configuration, they gain one degree of freedom, allowing the sliding joints to move freely. It should be noted that since the mechanisms are designed to track the knee joint movement, the main hinge joints $(\mathrm{R})$ will vary between $-10^{\circ}$ and $150^{\circ}$ only when attached to the human leg. Therefore, the only singular configuration that can occur in practice is when both prismatic joints are aligned on opposite sides of the pivot joint. Thus, only one unique singular configuration needs to be considered.

When the closed chain is not in this singular configuration, the main hinge joint of the mechanism automatically self-locates. This non-singular configuration is called the operating configuration and the special property of this type of mechanisms is called the property of self-adjustment. When the mechanism is in an operating configuration, the axis of rotation of the main hinge joint self-locates to align as closely as possible with the actual knee instantaneous axis of rotation as required by the support and guidance roles of the orthosis. The close coincidence of axes ensures the mechanical decoupling between the physiological movements and those prevented by the mechanism and, reciprocally, between the constraints associated with the physiological movements and the constraints imposed by the mechanism. This behavior is described in more detail below and analyzed in subsequent sections.

\subsection{Self-ajustement property}

Referring to Figs. 3 and 4 , let $b$ and $b^{\prime}$ be the distances between the knee axis and the segments of the mechanism. $b$ and $b^{\prime}$ are positive constants. Their values depend on the initial configuration of the closed chain. If $b \neq b^{\prime}$, the mechanism can never reach the position where $\theta_{2}=0$ and the mechanism can not be made singular. This situation is to be avoided. On the contrary, if $b=b^{\prime}, \theta_{2}$ can be equal to 0 . In this case, the mechanisms are in their singular configuration and the prismatic joints are free to slide, giving rise to internal movement. It is through this internal movement that the main hinge joint can relocate itself.

We can demonstrate that when internal movement is permitted, the mechanism is blocked. It is in this 
configuration that any torque applied by the user through the anatomical joint (modeled by $P_{1}$ or $P_{2}$ ) will create internal loading in the mechanism, which can be eliminated if an additional joint, for example at $D_{1}$ or $D_{2}$, is provided. These additional joints, modeled by spring-loaded hinges, represent the inevitable elasticity due to soft tissues present between the orthosis brace and the bone.

When loaded, these additional joints force the mechanisms to exit the singular configuration. Misalignment during operation is thus accompanied with an angular deflection of the additional joints which, in turn, generate a torque tending to move the main joint. We will demonstrate that if certain geometrical conditions are fulfilled, this torque will return the rotational joint of the PRP mechanism toward its operating position. We call this mode of action 'self-adjusting internal movement'.

As will be demonstrated in later sections, the pin joint of the horizontal PRP mechanism is located in the bisecting plane of the closed chain after self-adjustment, that is, when the chains are closed and not in a singular configuration. This can be stated as follows: for the first mechanism, the knee axis is in the symmetry plane when the following geometrical condition is verified ( $D_{1}^{\prime}$ being the projection of the point $P_{1}$ on the segment $C_{1} D_{1}$, see Fig. 3),

$$
\text { || } A_{1} B_{1}\|=b=\| C_{1} D_{1}^{\prime} \|=b^{\prime}
$$

As will be further demonstrated, the main hinge joint of the vertical PRP mechanism is coaxial with the knee axis after self-adjustement, provided vertical self-adjustment is performed after the horizontal one. Indeed the second mechanism operates only if the following geometrical conditions, which require that the horizontal self-adjustment be performed before the vertical adjustment, are verified $\left(D_{2}^{\prime}\right.$ denoting the projection of $P_{2}$ on $C_{2} D_{2}$ and $H_{1}$ and $H_{2}$ the projections of $P_{2}$ on linkages (1) and (2) of the mechanism, see Fig. 4),

$$
\left\|A_{2} P_{2}\right\|=\left\|B_{2} H_{1}\right\|, \quad\left\|P_{2} D_{2}^{\prime}\right\|=\left\|H_{2} C_{2}\right\|
$$

Moreover, the knee and mechanism axes coincide when the following conditions are verified:

$$
\begin{aligned}
& \left\|A_{2} B_{2}\right\|=\left\|D_{2}^{\prime} C_{2}\right\|=0, \\
& \left\|A_{2} P_{2}\right\|=\left\|B_{2} O_{2}\right\|, \\
& \left\|P_{2} D_{2}^{\prime}\right\|=\left\|O_{2} C_{2}\right\|
\end{aligned}
$$

The main hinge joint of the mechanism is thus located on these symmetry planes. However, in order to fulfill the above geometrical conditions, it is necessary that initially, during fixation onto the leg, the PRP mechanism is in its singular configuration.

Both vertical and horizontal adjustment mechanisms can obviously be integrated into a single mechanism as shown on Fig. 2, so as to obtain an automatic planar alignment of the joint axis passing through the point $O$ with the axis of the physiological joint. To create the horizontal self-adjustment motion, the two vertical translational joints $\left(\dot{r}_{v_{1}}\right.$ and $\left.\dot{r}_{v_{3}}\right)$ are blocked and the two horizontal translational joints $\left(\dot{r}_{h_{1}}\right.$ and $\dot{r}_{h_{3}}$ ) are freed and vice-versa for the vertical selfadjustment motion. This adaptation will be realized successively by releasing and blocking the prismatic joints of horizontal and vertical self adjustment mechanisms presented respectively in Sects. 3 and 4. Once the mechanism is well-adjusted onto the user's limb, and the orthosis' principal axis $\left(\dot{\theta}_{2}\right)$ is located as close as possible to the knee, all these DOFs are blocked so that the device can work in normal mode to protect the knee joint.

\subsection{Geometrical properties of PRP mechanisms}

When the first mechanism is in its operating configuration, the following property is verified (with $\alpha_{1}=\widehat{O_{1}} \widehat{P_{1}} H_{1}$ and $\alpha_{2}=\widehat{O_{1}} \widehat{P_{1}} H_{2}$, see Fig. 3):

$$
\frac{\sin \alpha_{1}}{\sin \alpha_{2}}=\frac{b}{b^{\prime}}
$$

- If $b=b^{\prime}$, then $\alpha_{1}=\alpha_{2}$. The line connecting both pin joints is the symmetry axis of the closed chain. The prismatic joints then slide in opposite directions, with the same speed. The angular velocities of the knee and the mechanism are equal $\left(\dot{\theta}_{2}=\dot{\theta}_{5}\right)$.

- If $b \neq b^{\prime}$, then the mechanism can never reach its singular position.

In the case of the second mechanism, the following properties are verified outside the singular configuration: $* *$

- The axes of rotation of the knee and the mechanism are perfectly aligned.

- Their angular velocities are equal $\left(\dot{\theta}_{2}=\dot{\theta}_{5}\right)$. 


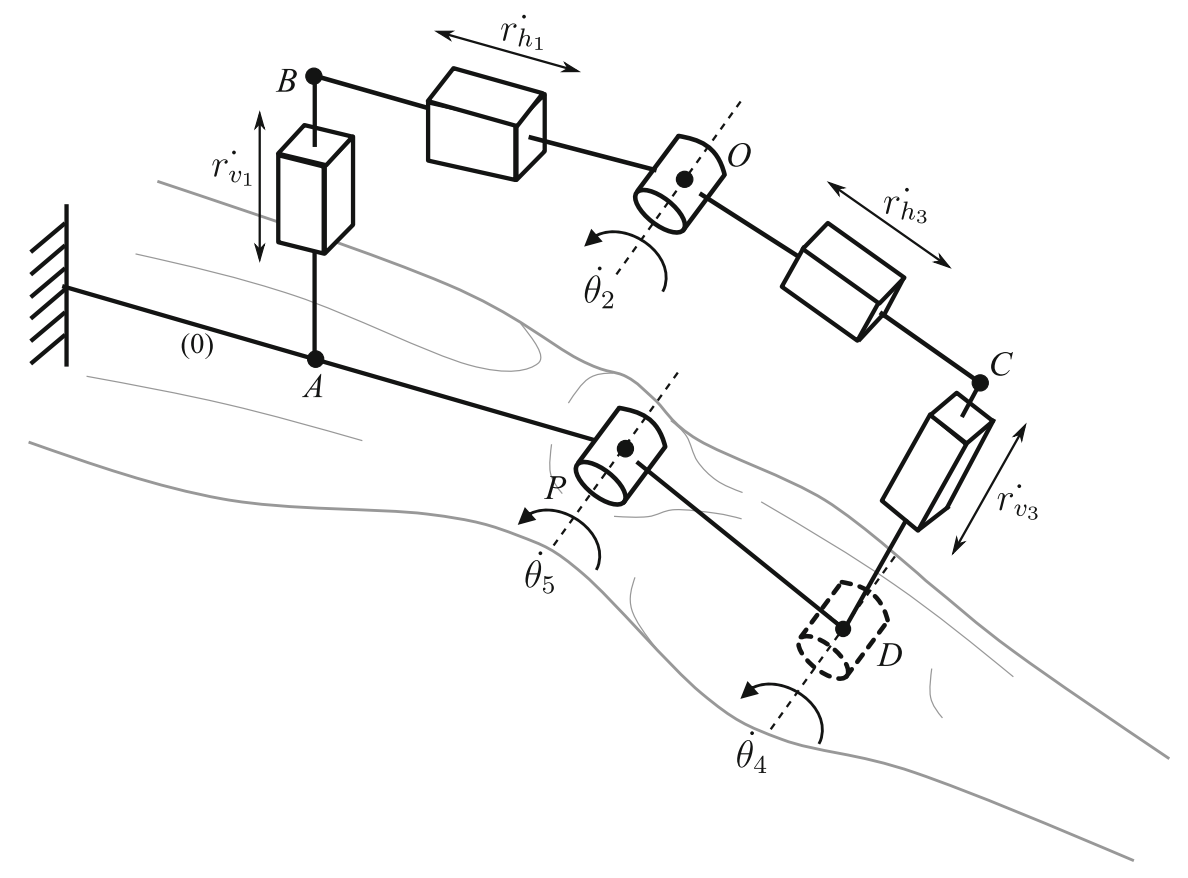

Fig. 2 Kinematic diagram showing the combination of the two adjustment mechanisms by horizontal and vertical prismatic joints (respectively $r_{h_{i}}$ and $r_{v_{i}}$ with $i=1,3$ ) allowing both horizontal and vertical self-adjustment. Subsequently, these variables will be denoted simply $r_{i}$ and $\dot{r}_{i}$. Segment $A P$ represents the thigh, segment $P D$ the calf. The knee is modeled as a pivot joint of angle $\theta_{5}$. The orthosis is composed of segments $A B, B O, O C$ and $C D . B O$ and $O C$ are articulated

- The velocities of the prismatic joints are equal to zero.

These properties are demonstrated in the following sections by connecting the PRP mechanisms to a simplified model of the knee joint. The analysis of the self-adjustment movements is then described in details and illustrated by simulations.

\section{Analysis of the horizontal self-adjustment movement}

\subsection{Modeling of the horizontal PRP mechanism}

The horizontal PRP mechanism is shown in Fig. 3 fixed onto the leg. For the purpose of this analysis, the knee joint is represented as a hinge. A pin joint and a torsional spring of stiffness $K$ are introduced at point $D_{1}$ to represent the torsional elasticity of the through a revolute joint R of angle $\theta_{2}$ passing through point $O$. The orthosis is rigidly attached to the thigh at point $A$ and through a spring of stiffness $K$ acting on a revolute joint of angle $\theta_{4}$ to the calf at point $D$. Prismatic joints between points $O$ and $B$, resp. $O$ and $C$ allow horizontal self-adjustment. Prismatic joints between points $A$ and $B$, resp. $D$ and $C$ allow vertical selfadjustment

attachments which can deform under constraints. With $\gamma=P_{1} \widehat{D_{1}} C_{1}=\pi / 2+\theta_{4}$ denoting the angle between the calf and the mechanism at $D_{1}$ and $\gamma_{0}$ its initial value when the PRP mechanism has just been attached, any angular variation at $D_{1}$ produces a torque caused by the deformation of the torsional spring, defined by:

$$
C_{D_{1}}=K\left(\gamma-\gamma_{0}\right)
$$

During its movement, the system will tend to minimize the elastic energy stored in the torsional spring. The torque generated by this spring will create the self-adjustment movement.

\subsection{Kinematic model of the horizontal PRP mechanism}

The loop-closure kinematic equations of the coupled system can be expressed at point $P_{1}$ as follows: 


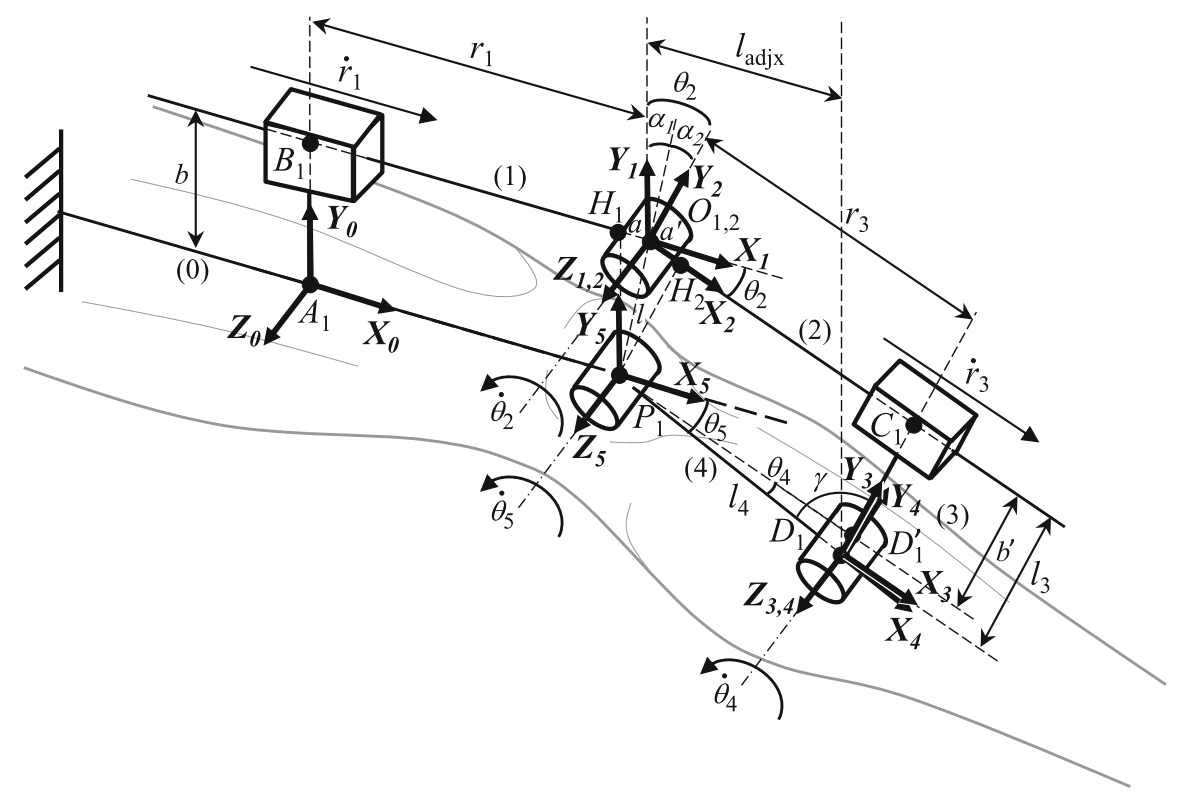

Fig. 3 Homokinetic PRP mechanism allowing a horizontal self-adjustment. Segment $A_{1} P_{1}$ represents the thigh, segment $P_{1} D_{1}$ the calf. The knee is modeled as a pivot joint of angle $\theta_{5}$. The orthosis is composed of segments $A_{1} B_{1}, B_{1} O_{1}, O_{1} C_{1}$ and $C_{1} D_{1} . B_{1} O_{1}$ and $O_{1} C_{1}$ are articulated through a revolute joint $\mathrm{R}$

$$
\boldsymbol{J}_{\boldsymbol{P}_{1}}(\boldsymbol{q}) \dot{\boldsymbol{q}}=0
$$

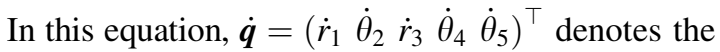
vector of the joints speeds of the complete mechanism (the anatomical joint plus the orthosis) and $\boldsymbol{J}_{\boldsymbol{P}_{\boldsymbol{I}}}(\boldsymbol{q})=$ $\left(\$_{1} \$_{2} \$_{3} \$_{4} \$_{5}\right)$ its Jacobian matrix expressed at point $P_{1}, \$_{i}$ being the unit twist of joint $i$ which can be written $\$_{i}=\left(\boldsymbol{x}_{\boldsymbol{i}} \boldsymbol{\theta}\right)^{\top}$ for a prismatic joint of axis $\boldsymbol{x}_{\boldsymbol{i}}$ and $\boldsymbol{\$}_{\boldsymbol{i}}=\left(\boldsymbol{P}_{\boldsymbol{l}} \boldsymbol{O}_{\boldsymbol{i}} \wedge z_{i} z_{i}\right)^{\top}$ for a revolute joint along axis $z_{i}$ passing through $O_{i}$ (here $\mathrm{O} 3=\mathrm{O} 4=\mathrm{D} 1$ and $\mathrm{O} 5=\mathrm{P} 1$, see Fig. 3).

\subsection{Constraints imposed during the attachment phase}

We assume the user standing still when the orthosis is attached to the leg, i.e. the knee joint at $P_{1}$ is fixed. Then if during the movement $\theta_{4}$ remains unchanged, of angle $\theta_{2}$ passing through point $O_{1}$. The orthosis is rigidly attached to the thigh at point $A_{1}$ and through a spring of stiffness $K$ acting on a revolute joint of angle $\gamma$ to the calf at point $D_{1}$. Prismatic joints at points $B_{1}$ and $C_{1}$ allow horizontal selfadjustment. See the text for more details

the distance $b^{\prime}$ between $P_{1}$ and the segment $O_{1} C_{1}$ $\left(b^{\prime}=C_{1} D_{1}^{\prime}\right)$ will be constant. The distance $b=A_{1} B_{1}$ between $P_{1}$ and the segment $O_{1} B_{1}$ being also constant, if $b \neq b^{\prime}$, the mechanism cannot fully extend (i.e. segment $O_{1} B_{1}$ aligned with $O_{1} C_{1}$ ) if the attachment at point $D_{1}$ does not deform. Distances $b$ and $b^{\prime}$ must be equal to avoid this phenomenon. The PRP mechanism has to be in a singular configuration $\left(\theta_{2}=0\right)$ during its fixation on the thigh and calf (segments $P_{1} A_{1}$ and $\left.P_{1} D_{1}\right)$ to respect this geometrical condition.

\subsection{Analysis of the singular configuration}

To analyse the behaviour of the proposed mechanism, we compute the Jacobian matrix of the closed loop mechanism shown on Fig. 3 at point $P_{1}$ in the frame $R_{0}=\left(\boldsymbol{A}_{\mathbf{l}}, \boldsymbol{x}_{\boldsymbol{0}}, \boldsymbol{y}_{\mathbf{0}}\right)$ :

$$
\boldsymbol{J}_{\boldsymbol{P}_{1}}(\boldsymbol{q})_{/_{R_{0}}}=\left(\begin{array}{ccccc}
1 & \cos \theta_{2}\left(l_{3}+l_{4} \sin \theta 4\right)-\sin \theta_{2}\left(r_{3}-l_{4} \cos \theta_{4}\right) & \cos \theta_{2} & l_{4} \sin \left(\theta_{2}+\theta_{4}\right) & 0 \\
0 & \sin \theta_{2}\left(l_{3}+l_{4} \sin \theta 4\right)+\cos \theta_{2}\left(r_{3}-l_{4} \cos \theta_{4}\right) & \sin \theta_{2} & -l_{4} \cos \left(\theta_{2}+\theta_{4}\right) & 0 \\
0 & 1 & 0 & 1 & 1
\end{array}\right)
$$


Knowing that $\left(l_{3}+l_{4} \sin \theta 4\right)=b^{\prime}$ and $r_{3}-$ $l_{4} \cos \theta_{4}=a^{\prime}$ and that $b^{\prime} \cos \theta_{2}-a^{\prime} \sin \theta_{2}=b$ and $b^{\prime} \sin \theta_{2}+a^{\prime} \cos \theta_{2}=-a$ (with $(a, b)$ the components of vector $\boldsymbol{P}_{\boldsymbol{1}} \boldsymbol{O}_{\boldsymbol{I}}$ in the frame $R_{0}$ ), we obtain:

$\boldsymbol{J}_{\boldsymbol{P}_{1}}(\boldsymbol{q})_{\text {R }_{0}}=\left(\begin{array}{ccccc}1 & b & \cos \theta_{2} & l_{4} \sin \left(\theta_{2}+\theta_{4}\right) & 0 \\ 0 & -a & \sin \theta_{2} & -l_{4} \cos \left(\theta_{2}+\theta_{4}\right) & 0 \\ 0 & 1 & 0 & 1 & 1\end{array}\right)$

When $\theta_{2}=\dot{\theta}_{2}=0$, the loop-closure kinematic system of equations at point $P_{1}$ can be written as follows:

$\left\{\begin{array}{l}\dot{r}_{1}+\dot{r}_{3}+\dot{\theta}_{4} l_{4} \sin \theta_{4}=0 \\ -\dot{\theta}_{4} l_{4} \cos \theta_{4}=0 \\ \dot{\theta}_{4}=-\dot{\theta}_{5}\end{array}\right.$

Assuming that $l_{4} \neq 0$, we get $\dot{\theta}_{4}=0$ and $\dot{\theta}_{5}=0$ ( $\cos \theta_{4} \neq 0$ as $\theta_{4}$ is a small angle). The mechanism is in a singular configuration and the knee cannot move. On the other hand, $\dot{r}_{1}=-\dot{r}_{3}$. Both prismatic joints can slide at the same speed. An internal movement appears where the orthosis can move freely in parallel with the leg.

\subsection{Analysis of the self-adjustment movement}

For the analysis of the self-adjustement property, we compute the Jacobian matrix at point $D_{1}$ in the frame $R_{2}=\left(\boldsymbol{O}_{2}, \boldsymbol{x}_{2}, \boldsymbol{y}_{2}\right)$. With $r_{3}$ and $-l_{3}$ the components of the vector $\boldsymbol{O}_{1} \boldsymbol{D}_{\boldsymbol{1}}$ written in the frame $R_{2}$, the Jacobian matrix becomes:

$\boldsymbol{J}_{\boldsymbol{D}_{1}}(\boldsymbol{q})_{/_{R_{2}}}=\left(\begin{array}{ccccc}\cos \theta_{2} & l_{3} & 1 & 0 & -l_{4} \sin \theta_{4} \\ -\sin \theta_{2} & r_{3} & 0 & 0 & l_{4} \cos \theta_{4} \\ 0 & 1 & 0 & 1 & 1\end{array}\right)$

\subsubsection{Self-adjustment at fixed knee position}

We first assume that the knee is fixed $\left(\dot{\theta}_{5}=0\right)$, i.e. we make the analysis at a given leg position. With this assumption, the last column of $\boldsymbol{J}_{\boldsymbol{D}_{\boldsymbol{I}}}(\boldsymbol{q})_{/_{R_{2}}}$ vanishes and the loop-closure kinematic equations at point $D_{1}$ can be expressed as follows:

$$
\left\{\begin{array}{l}
\dot{r}_{1} \cos \theta_{2}+l_{3} \dot{\theta}_{2}+\dot{r}_{3}=0 \\
-\dot{r}_{1} \sin \theta_{2}+r_{3} \dot{\theta}_{2}=0 \\
\dot{\theta}_{2}=-\dot{\theta}_{4}
\end{array}\right.
$$

- When $\theta_{2}=0$, if $r_{3} \neq 0$ then $\dot{\theta}_{2}=\dot{\theta}_{4}=0$ and $\dot{r}_{1}=-\dot{r}_{3}$. The main hinge joint cannot move. However, as was established in Sect. 3.4, an internal mobility appears and the two prismatic joints can slide freely if external forces, as for example the gravity, apply.

- When $\theta_{2} \neq 0$, we get (with $l_{a d j x}=r_{3} \cos \theta_{2}+$ $l_{3} \sin \theta_{2}$ the coordinate of the vector $\boldsymbol{O}_{\boldsymbol{1}} \boldsymbol{D}_{\boldsymbol{1}}$ along $\left.x_{0}\right)$ :

$$
\dot{r}_{1}=\frac{r_{3}}{\sin \theta_{2}} \dot{\theta}_{2}, \quad \dot{r}_{3}=-\frac{l_{a d j x}}{\sin \theta_{2}} \dot{\theta}_{2}
$$

The speeds $\dot{r}_{1}$ and $\dot{r}_{3}$ have opposite signs, which means that the sliders move in the same sense, leading the mechanism to the equilibrium position in which $\dot{\theta}_{4}=0$. The larger $r_{3}$ and $l_{a d j x}$, the faster the adjustement.

As can be seen from Fig. 3, $\theta_{4}$ is negative and will thus increase towards 0 due to the spring torque, i.e. $\dot{\theta}_{4}>0$. As a consequence, we deduce from eq. 11 that $\dot{\theta}_{2}<0$, and from eq. 12 that $\dot{r}_{1}>0$ and $\dot{r}_{3}<0$ (since $\theta_{2}$ is also negative). Distance $a$, which is smaller than $a^{\prime}$, will increase (the orthosis will move to the right). When the spring is back to its neutral position, we have $\dot{\theta}_{4}=0$ thus $\dot{\theta}_{2}=0, \dot{r}_{1}=$ 0 (outside the singular configuration) and $\dot{r}_{3}=0$. The self-adjustment movement stops.

\subsubsection{Self-adjustment with moving knee}

When $\theta_{5} \neq 0$, the loop-closure kinematic equations are:

$$
\left\{\begin{array}{l}
\dot{r}_{1} \cos \theta_{2}+l_{3} \dot{\theta}_{2}+\dot{r}_{3}=l_{4} \sin \theta_{4} \dot{\theta}_{5} \\
-\dot{r}_{1} \sin \theta_{2}+r_{3} \dot{\theta}_{2}=-l_{4} \cos \theta_{4} \dot{\theta}_{5} \\
\dot{\theta}_{2}=-\dot{\theta}_{4}-\dot{\theta}_{5}
\end{array}\right.
$$

Thus we get:

$$
\begin{aligned}
& \dot{r}_{1}=\frac{-r_{3}}{\sin \theta_{2}} \dot{\theta}_{4}+\frac{-r_{3}+l_{4} \cos \theta_{4}}{\sin \theta_{2}} \dot{\theta}_{5}, \\
& \dot{r}_{3}=\frac{r_{3}+l_{3} \tan \theta_{2}}{\tan \theta_{2}} \dot{\theta}_{4} \\
& +\frac{\left(r_{3}-l_{4} \cos \theta_{4}+l_{3} \tan \theta_{2}+l_{4} \sin \theta_{4} \tan \theta_{2}\right)}{\tan \theta_{2}} \dot{\theta}_{5}
\end{aligned}
$$


Knowing that $r_{3}-l_{4} \cos \theta_{4}=a^{\prime}, \quad\left(r_{3}+l_{3}\right.$ $\left.\tan \theta_{2}\right) \cos \theta_{2}=l_{a d j x}, l_{3}+l_{4} \sin \theta_{4}=b^{\prime}$ and $\left(a^{\prime}+b^{\prime}\right.$ $\left.\tan \theta_{2}\right) \cos \theta_{2}=-a$ (see Fig. 3), we obtain:

$$
\dot{r}_{1}=\frac{-r_{3}}{\sin \theta_{2}} \dot{\theta}_{4}-\frac{a^{\prime}}{\sin \theta_{2}} \dot{\theta}_{5}, \quad \dot{r}_{3}=\frac{l_{a d j x}}{\sin \theta_{2}} \dot{\theta}_{4}-\frac{a}{\sin \theta_{2}} \dot{\theta}_{5}
$$

- We can see from eq. 16 that the knee flexion $\left(\dot{\theta}_{5}>0\right)$ tends to move the sliders in opposite directions ( $\dot{\theta}_{5}$ generates rotations of $r_{1}$ and $r_{3}$ of the same sign). This phenomenom remains after selfadjustment (see below).

- During the knee flexion $\left(\dot{\theta}_{5}>0\right)$, we can see from the last equation of system 13 that both $\theta_{2}$, the orthosis angle, and/or $\theta_{4}$, the spring angle at the calf attachmnent, change. If the orthosis is not in the bissecting plane of the leg, it is obvious that for a given value of $r_{1}$, the orthosis can rotate only if the calf attachment also rotates. As a consequence, the spring loads up and the induced torque tends to move both sliders in the same direction $\left(\dot{\theta}_{4}\right.$ generates $r_{1}$ and $r_{3}$ rotations of opposite signs according to eq. 16).

Referring to Fig. 3 again, $\theta_{4}$ is negative and will thus increase towards 0 due to the spring torque, i.e. $\dot{\theta}_{4}>0$. As a consequence, the spring action will tend to increase $r_{1}$ and decrease $r_{3}\left(\theta_{2}\right.$ is negative). Distance $a$, which is smaller than $a^{\prime}$, will increase until $a=a^{\prime}$, i.e. the orthosis lies in the symetry plane of the leg. If $a=a^{\prime}$, the orthosis can rotate without modifying the spring angle (whose stiffness prevents rotations at calf attachment in this configuration). In other words, we will have $\dot{\theta}_{4}=0$ and $\dot{r}_{1}=\dot{r}_{3}$. The orthosis will remain in the symetry plane of the leg (see next section).

\subsection{Kinematic analysis of the mechanism after the self-adjustment movement}

Assuming that the resistance of the torsional spring at $D_{1}$ prevents large motions at the calf attachment after self-adjustment, segments $P_{1} D_{1}$ and $D_{1} C_{1}$ can be considered rigidly connected and $\theta_{4}$ fixed at a constant value. The Jacobian matrix written at point $P_{1}$ in the frame $R_{2}$ is:
$\boldsymbol{J}_{\boldsymbol{P}_{1}}(\boldsymbol{q})_{/_{R_{2}}}=\left(\begin{array}{cccc}\cos \theta_{2} & l_{3}+l_{4} \sin \theta_{4} & 1 & 0 \\ -\sin \theta_{2} & r_{3}-l_{4} \cos \theta_{4} & 0 & 0 \\ 0 & 1 & 0 & 1\end{array}\right)$

Knowing that $l_{3}+l_{4} \sin \theta_{4}=b^{\prime}$ and $r_{3}-l_{4}$ $\cos \theta_{4}=a^{\prime}$, the loop-closure at $P_{1}$ provides the following system of equations:

$\left\{\begin{array}{l}\dot{r}_{1} \cos \theta_{2}+b^{\prime} \dot{\theta}_{2}+\dot{r}_{3}=0 \\ -\dot{r}_{1} \sin \theta_{2}+a^{\prime} \dot{\theta}_{2}=0 ; l \\ \dot{\theta}_{2}=-\dot{\theta}_{5}\end{array}\right.$

The linear velocities of the two prismatic joints can hence be computed as:

$\dot{r}_{1}=\frac{a^{\prime}}{\sin \theta_{2}} \dot{\theta}_{2}, \quad \dot{r}_{3}=\frac{-a^{\prime} \cos \theta_{2}-b^{\prime} \sin \theta_{2}}{\sin \theta_{2}} \quad \dot{\theta}_{2}=\frac{a}{\sin \theta_{2}} \dot{\theta}_{2}$

Thus, knowing that after auto-ajustment $a=a^{\prime}$, we obtain finally:

$\dot{r}_{3}=\dot{r}_{1}$

The velocities of the prismatic joints are equal. They move in opposite senses at the same speed and $P_{1}$ remains at the symmetry axis of the mechanism. It is worth noting that if $a=a^{\prime}$ tends to zero, i.e. if the main hinge joint of the orthosis is close to the knee axis, the movements of the sliders become negligible as $\dot{r}_{3}$ and $\dot{r}_{1}$ also tend to zero.

\section{Analysis of the vertical self-adjustment movement}

\subsection{Modeling of the vertical PRP mechanism}

The vertical PRP mechanism is shown in Fig. 4. It is composed of a main hinge joint and two prismatic joints orthogonal to segments $\mathrm{B}_{2} \mathrm{O}_{2}$ and $\mathrm{O}_{2} \mathrm{C}_{2}$. We assume that the system is already adjusted horizontally and we consider that segments $H_{1} B_{2}$ and $P_{2} A_{2}$ are equal, that is:

$H_{1} B_{2}=P_{2} A_{2}=l_{1}$

An elastic element of stiffness $K$ is introduced at point $D_{2}$ to simulate the flexibility of the attachment. We denote $\gamma=P_{2} \widehat{D_{2}} C_{2}=\pi / 2+\theta_{4}$ the angle 


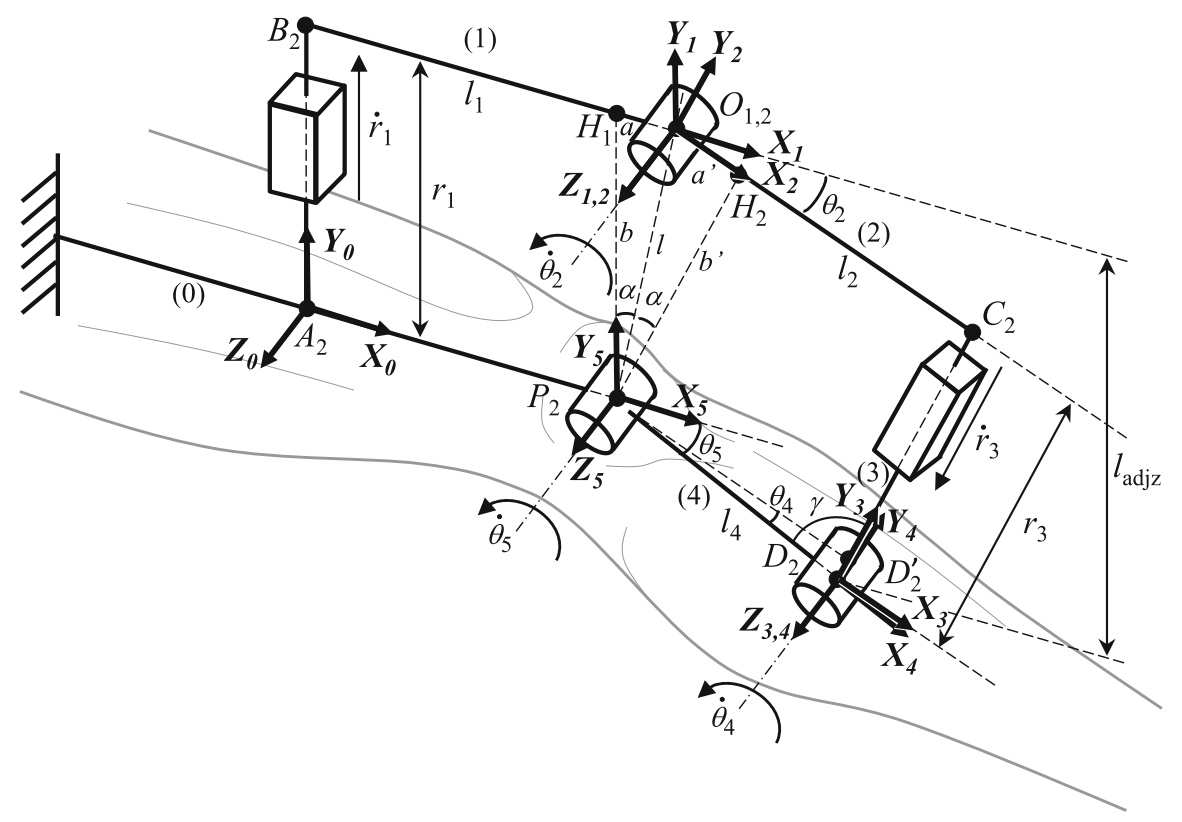

Fig. 4 Homokinetic PRP mechanism allowing a vertical selfadjustment. Segment $A_{2} P_{2}$ represents the thigh, segment $P_{2} D_{2}$ the calf. The knee is modeled as a pivot joint of angle $\theta_{5}$. The orthosis is composed of segments $A_{2} B_{2}, B_{2} O_{2}, O_{2} C_{2}$ and $C_{2} D_{2}$. $\mathrm{B}_{2} \mathrm{O}_{2}$ and $\mathrm{O}_{2} \mathrm{C}_{2}$ are articulated through a revolute joint $\mathrm{R}$ of

between the calf and the mechanism and $\gamma_{0}$ its initial value obtained when the orthosis is in its initial position where $\theta_{2}=0$. The spring at $D_{2}$ is at equilibrium in this configuration.

\subsection{Kinematic model of the vertical PRP mechanism}

As for the horizontal PRP mechanism, for the purpose of this analysis, we write the Jacobian matrix of the closed loop system composed of the leg and orthosis in the base frame $R_{0}=\left(\boldsymbol{A}_{2}, \boldsymbol{x}_{\boldsymbol{0}}, \boldsymbol{y}_{\boldsymbol{0}}\right)$ at point $P_{2}$ and in the frame $R_{2}=\left(\boldsymbol{O}_{2}, \boldsymbol{x}_{2}, \boldsymbol{y}_{2}\right)$ at point $D_{2}$. These equations will be clarified in the foregoing. angle $\theta_{2}$ passing through point $O_{2}$. The orthosis is rigidly attached to the thigh at point $A_{2}$ and through a spring of stiffness $K$ acting on a revolute joint of angle $\gamma$ to the calf at point $D_{2}$. Prismatic joints between points $A_{2}$ and $B_{2}$, resp. $D_{2}$ and $C_{2}$ allow vertical self-adjustment. See the text for more details

\subsection{Mechanical constraint in the attachment phase}

Initially, the mechanism is necessarily located in the configuration in which the segments $P_{2} A_{2}$ and $P_{2} D_{2}^{\prime}$ are aligned. If these segments are in flexion at that moment, the mechanism can not enter its singular configuration later. For example if the segment $\mathrm{O}_{2} \mathrm{C}_{2}$ is longer than $P_{2} D_{2}^{\prime}$, it will prevent the mechanism to enter its singular configuration where $\mathrm{O}_{2} \mathrm{C}_{2}=\mathrm{P}_{2} \mathrm{D}_{2}^{\prime}$.

\subsection{Analysis of the singular configuration}

To analyse the behaviour of the vertical PRP mechanism, we compute the Jacobian matrix at point $P_{2}$ in the frame $R_{0}=\left(\boldsymbol{A}_{2}, \boldsymbol{x}_{\boldsymbol{0}}, \boldsymbol{y}_{\mathbf{0}}\right)$ :

$\boldsymbol{J}_{\boldsymbol{P}_{2}}(\boldsymbol{q})_{/_{R_{0}}}=\left(\begin{array}{ccccc}0 & \cos \theta_{2}\left(r_{3}+l_{4} \sin \theta 4\right)-\sin \theta_{2}\left(l_{2}-l_{4} \cos \theta_{4}\right) & \sin \theta_{2} & l_{4} \sin \left(\theta_{2}+\theta_{4}\right) & 0 \\ 1 & \sin \theta_{2}\left(r_{3}+l_{4} \sin \theta 4\right)+\cos \theta_{2}\left(l_{2}-l_{4} \cos \theta_{4}\right) & -\cos \theta_{2} & -l_{4} \cos \left(\theta_{2}+\theta_{4}\right) & 0 \\ 0 & 1 & 0 & 1 & 1\end{array}\right)$ 
Knowing that $\left(r_{3}+l_{4} \sin \theta 4\right)=b^{\prime}$ and $l_{2}-$ $l_{4} \cos \theta_{4}=a^{\prime}$ and that $b^{\prime} \cos \theta_{2}-a^{\prime} \sin \theta_{2}=b$ and $b^{\prime} \sin \theta_{2}+a^{\prime} \cos \theta_{2}=-a$ ( $a$ and $b$ being the components of vector $\boldsymbol{P}_{2} \boldsymbol{O}_{2}$ in the frame $R_{0}$ ), this equation can be written in a simplified form as follows:

$$
\begin{aligned}
& \boldsymbol{J}_{\boldsymbol{P}_{2}}(\boldsymbol{q})_{/ R_{0}} \\
& =\left(\begin{array}{ccccc}
0 & b & \sin \theta_{2} & l_{4} \sin \left(\theta_{2}+\theta_{4}\right) & 0 \\
1 & -a & -\cos \theta_{2} & -l_{4} \cos \left(\theta_{2}+\theta_{4}\right) & 0 \\
0 & 1 & 0 & 1 & 1
\end{array}\right)
\end{aligned}
$$

When $\theta_{2}=\dot{\theta}_{2}=0$, the system of loop-closure kinematic equations can be written:

$$
\left\{\begin{array}{l}
\dot{\theta}_{4} l_{4} \sin \theta_{4}=0 \\
\dot{r}_{1}-\dot{r}_{3}-\dot{\theta}_{4} l_{4} \cos \theta_{4}=0 \\
\dot{\theta}_{4}=-\dot{\theta}_{5}
\end{array}\right.
$$

It is worth noticing that knee movements produce similar calf attachment displacements $\left(\dot{\theta}_{4}=-\dot{\theta}_{5}\right)$. Thus assuming that $l_{4} \neq 0, l_{4} \sin \theta_{4}$ will not remain null even if it is momentarily and we assume that $l_{4} \sin \theta_{4} \neq 0$. In this case $\dot{\theta}_{4}=\dot{\theta}_{5}=0$ and $\dot{r}_{1}=\dot{r}_{3}$. The mechanism is in a singular configuration and the knee can not move. On the other hand, both prismatic joints slide in the same direction. An internal movement appears where the orthosis can move freely perpendicular to the thigh.

\subsection{Analysis of self-adjustment movement}

To analyse the self-adjustment property, we compute the Jacobian matrix of the complete mechanism (composed of the leg and the orthosis) at point $D_{2}$ in the frame $R_{2}=\left(\boldsymbol{O}_{2}, \boldsymbol{x}_{2}, \boldsymbol{y}_{2}\right)$ :

$\boldsymbol{J}_{\boldsymbol{D}_{2}}(\boldsymbol{q})_{/_{R_{2}}}=\left(\begin{array}{ccccc}\sin \theta_{2} & r_{3} & 0 & 0 & -l_{4} \sin \theta_{4} \\ \cos \theta_{2} & l_{2} & -1 & 0 & l_{4} \cos \theta_{4} \\ 0 & 1 & 0 & 1 & 1\end{array}\right)$

\subsubsection{Self-adjustment at fixed knee position}

As for the horizontal mechanism, we first assume that the self-adjustment occurs around a given leg position, i.e. that the knee is fixed $\left(\dot{\theta}_{5}=0\right)$. This hypothesis remains valid for an infinitesimal motion around the current knee angle if the leg is moving. Under this assumption, the last column of $\boldsymbol{J}_{\boldsymbol{D}_{2}}(\boldsymbol{q})_{\mathrm{R}_{2}}$ disappears and the system of loop-closure kinematic equations are:

$$
\left\{\begin{array}{l}
\dot{r}_{1} \sin \theta_{2}+r_{3} \dot{\theta}_{2}=0 \\
\dot{r}_{1} \cos \theta_{2}+l_{2} \dot{\theta}_{2}-\dot{r}_{3}=0 \\
\dot{\theta}_{2}=-\dot{\theta}_{4}
\end{array}\right.
$$

With $l_{a d j z}=r_{3} \cos \theta_{2}-l_{2} \sin \theta_{2}$ the component of the vector $\boldsymbol{O}_{2} \boldsymbol{D}_{2}$ along $\boldsymbol{y}_{\boldsymbol{0}}$, we get:

$$
\dot{r}_{1}=-\frac{r_{3}}{\sin \theta_{2}} \dot{\theta_{2}} \quad \dot{r}_{3}=-\frac{l_{a d j z}}{\sin \theta_{2}} \dot{\theta}_{2}
$$

The prismatic joints slide in the same direction as $\dot{r}_{1}$ and $\dot{r}_{3}$ are of the same sign. To illustrate this phenomenom, we refer again to Fig. 4. As $\theta_{4}<0$, the spring at $D_{2}$ produces a torque which tends to cancel $\theta_{4}$, hence $\dot{\theta}_{4}>0$. From the last equation of system 26 , we deduce that $\dot{\theta}_{2}<0$ and from equation 27 that $\dot{r}_{1}$ and $\dot{r}_{3}$ are negative $\left(\sin \theta_{2}<0\right)$. Both prismatic joints will slide towards the leg, i.e. the main hinge joint $z_{2}$ will get closer to the knee axis $z_{5}$. When the spring is back to its neutral position, $\dot{\theta}_{4}=0$, thus $\dot{\theta}_{2}=0, \quad \dot{r}_{1}=0$ and $\dot{r}_{3}=0$ (outside the singular configuration). The self-adjustment movement stops. The same is true when $O_{2}$ and $P_{2}$ are coincident as $r_{3}=\dot{r}_{3}=0$ in this case, thus $\dot{r}_{1}=0$ (outside the singular configuration).

\subsubsection{Self-adjustment with moving knee}

When $\theta_{5} \neq 0$, the loop-closure system of kinematic equations of the system at point $D_{2}$ are:

$$
\left\{\begin{array}{l}
\dot{r}_{1} \sin \theta_{2}+r_{3} \dot{\theta}_{2}=l_{4} \sin \theta_{4} \dot{\theta}_{5} \\
\dot{r}_{1} \cos \theta_{2}+l_{2} \dot{\theta}_{2}-\dot{r}_{3}=-l_{4} \cos \theta_{4} \dot{\theta}_{5} \\
\dot{\theta}_{2}=-\dot{\theta}_{4}-\dot{\theta}_{5}
\end{array}\right.
$$

Thus we get:

$$
\begin{aligned}
\dot{r}_{1}= & \frac{r_{3}}{\sin \theta_{2}} \dot{\theta}_{4}+\frac{r_{3}+l_{4} \sin \theta_{4}}{\sin \theta_{2}} \dot{\theta}_{5}, \\
\dot{r}_{3}= & \frac{r_{3}-l_{2} \tan \theta_{2}}{\tan \theta_{2}} \dot{\theta}_{4} \\
& +\frac{\left(r_{3}+l_{4} \sin \theta_{4}-l_{2} \tan \theta_{2}+l_{4} \cos \theta_{4} \tan \theta_{2}\right)}{\tan \theta_{2}} \dot{\theta}_{5}
\end{aligned}
$$

We can see on Fig. 4 that $r_{3}+l_{4} \sin \theta_{4}=b^{\prime}$, $\left(r_{3}-l_{2} \tan \theta_{2}\right) \cos \theta_{2}=l_{a d j z}, \quad l_{2}-l_{4} \cos \theta_{4}=a^{\prime}$ and 


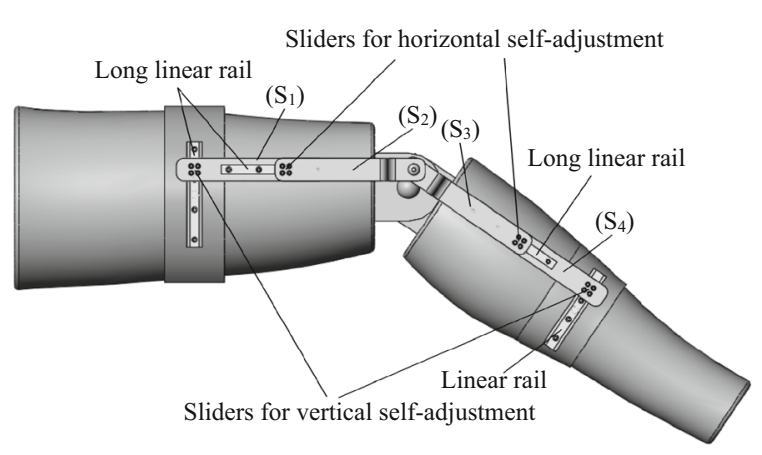

Fig. 5 The simulated self-adjustment mechanism

$\left(b^{\prime}-a^{\prime} \tan \theta_{2}\right) \cos \theta_{2}=b$. Previous equations can thus be written:

$$
\dot{r}_{1}=\frac{r_{3}}{\sin \theta_{2}} \dot{\theta}_{4}+\frac{b^{\prime}}{\sin \theta_{2}} \dot{\theta}_{5}, \quad \dot{r}_{3}=\frac{l_{a d j z}}{\sin \theta_{2}} \dot{\theta}_{4}+\frac{b}{\sin \theta_{2}} \dot{\theta}_{5}
$$

Both knee movements $\left(\dot{\theta}_{5} \neq 0\right)$ and spring action ( $\dot{\theta}_{4} \neq 0$ due to the spring torque) produce movements of the sliders in the same direction. Refering to Fig. 4, $\theta_{5}$ increases as the knee is bent, i.e. $\dot{\theta}_{5}>0$. Also $\theta_{4}<0$ and will tend to increase towards its initial value due to the spring torque, i.e. $\dot{\theta}_{4}>0$. Thus both $\dot{r}_{1}<0$ and $\dot{r}_{3}<0\left(\sin \theta_{2}<0\right)$ and both prismatic joints slide in the same direction towards the leg.

\section{Simulation of the self-adjustement mechanisms}

Previous theoretical results are verified numerically using the dynamics simulation software SolidWorks Motion Analysis. The considered mechanism is shown in Fig. 5 and the mass properties of the mechanical parts are given in Table 1. A pin joint is introduced at the calf attachment of the leg to take into account the attachment's flexibility. We suppose, however, that the mechanism is fixed rigidly to the thigh. In order to initiate the horizontal self-adjustment movement, the two vertical sliders are blocked. Then once the mechanism is aligned in the horizontal direction, the two horizontal sliders are blocked in return and the two vertical sliders are released in order to create the vertical self-adjustment movement. It is noticed that the knee must fully extended (at $0^{\circ}$ ) before switching between the horizontal and the vertical self-adjustment motions.

Figure 6 displays the results of the simulation of the horizontal self-adjustment motion. The knee joint is set to move from $0^{\circ}$ to $-120^{\circ}$ during 1 second, the stiffness of the calf attachment at $1 \mathrm{~N} . \mathrm{m} /{ }^{\circ}$ with a damper coefficient of $\left.100 \mathrm{~N} . \mathrm{m} / \mathrm{C}^{\circ} / \mathrm{s}\right)$, and the friction coefficient of the two sliders at 0.01 . The selfadjustment movement takes place at approximately $17^{\circ}$ in flexion. Large displacements occur in the two sliders and lead the mechanism to its equilibrium position. Then, they move slowly at the same speed. The angular velocity of the orthosis fluctuates during the self-adjustment movement and becomes equal to the knee joint's velocity once the mechanism is readjusted.

The results of the vertical self-adjustment are displayed on Fig. 7. We make the hypothesis that the orthosis is already adjusted in the horizontal direction. The first attachment is supposed to be rigid and a torsional spring is added at the calf attachment. As in the case of horizontal self-adjustment, the knee joint is set to move from $0^{\circ}$ to $-120^{\circ}$ during 1 second, the stiffness of the calf attachment at $1 \mathrm{~N} . \mathrm{m} /{ }^{\circ}$ with a damper coefficient of $\left.100 \mathrm{~N} . \mathrm{m} / \mathrm{(}^{\circ} / \mathrm{s}\right)$, and the friction coefficient of the two sliders at 0.01 .

The self-adjustment occurs when the torque in the spring reaches 4.1 N.m. This is much higher than the spring's torque of the horizontal self-adjusting mechanism, which means that the vertical self-adjustment is more difficult to obtain than the horizontal one. This can be explained by the fact that, in the vertical selfadjustment simulation, the force generated by the calf attachment (which is modeled as a torsional spring) is nearly perpendicular to the direction of movement of the first slider joint. It makes it difficult to create the

Table 1 Dimension and mass property of the mechanical parts

\begin{tabular}{lllllll}
\hline & Long rail & Rail & $\left(S_{1}\right)$ & $\left(S_{2}\right)$ & $\left(S_{3}\right)$ & $\left(S_{4}\right)$ \\
\hline Size $(\mathrm{mm})$ & $10 \times 2 \times 140$ & $10 \times 12 \times 120$ & $5 \times 30 \times 200$ & - & - & $5 \times 30 \times 220$ \\
Mass $(\mathrm{g})$ & 114.5 & 97.2 & 234.5 & 257.3 & 257.9 & 258.5 \\
$M_{z z}\left(\mathrm{~kg} \mathrm{~mm}{ }^{2}\right)$ & 185.9 & 118.2 & 774.9 & 807.2 & 808.4 & 1031.8 \\
\hline
\end{tabular}


Fig. 6 Horizontal selfadjustment of the PRP mechanism. Top: PRP mechanism in different representative

configurations. a Initial configuration. b

Configuration before selfadjustment. c Configuration just after self adjustment. d Final configuration after complete knee flexion. Bottom (1) Orthosis angle $\left(\theta_{2}\right)$. (2) Orthosis angular velocity $\left(\dot{\theta}_{2}\right)$. (3)

Displacement of the sliders 1 and 3. (4) Velocity of the sliders 1 and 3. (5) Angle at the calf attachment $\left(\theta_{4}\right)$. (6) Torque at the calf attachment. The horizontal axis represents the angle of the knee joint

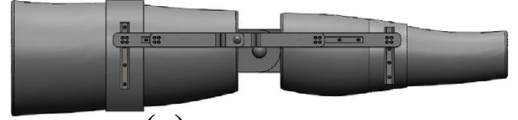

(a)
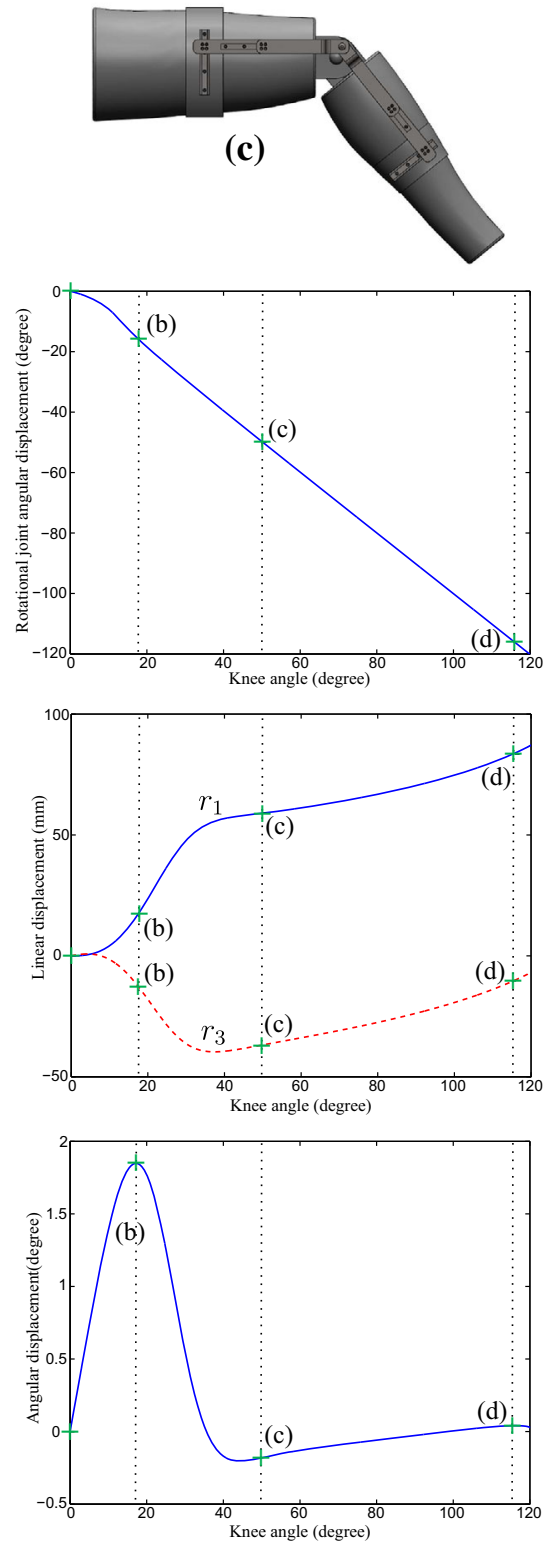

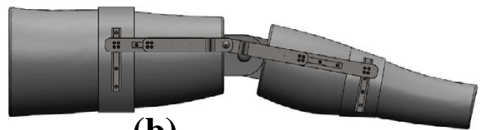

(b)
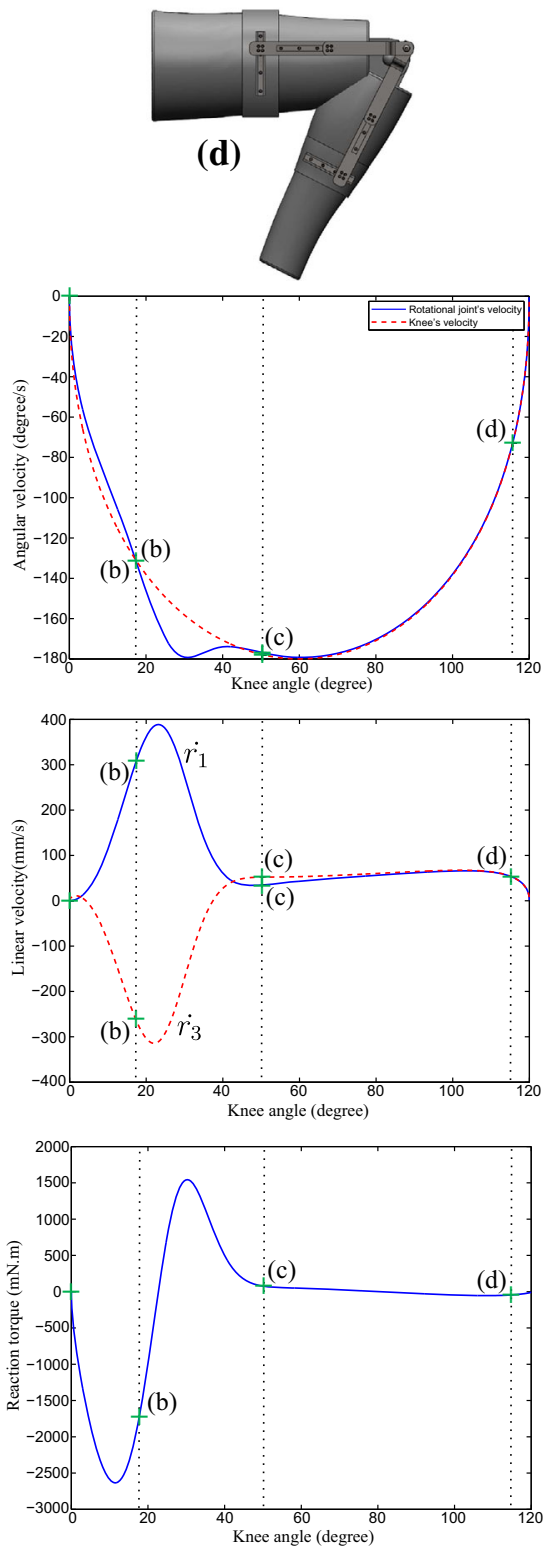

self-adjustment movement. On the contrary, in the horizontal self-adjustment simulation, this force component is transmitted nearly in the direction of movement of the two sliders, thus facilitating the self-adjustment movement.

\section{Discussion and conclusion}

In this paper, novel mechanisms which can self-adjust to a supporting structure, i.e. self-align with its joints, are proposed. Such mechanisms are particularly well 
Fig. 7 Simulation results of the vertical self-adjustment movement. Top: PRP mechanism in different representative configurations. a Initial configuration. b

Configuration during the beginning of selfadjustment. c Configuration after self adjustment. d Final configuration after complete knee flexion. Bottom: (1) Orthosis angle $\left(\theta_{2}\right) .(2)$ Orthosis angular velocity $\left(\dot{\theta}_{2}\right)$. (3) Displacement of the sliders 1 and 3. (4) Velocity of the sliders 1 and 3. (5) Angle at the calf attachment $\left(\theta_{4}\right)$. (6) Torque at the calf attachment. The horizontal axis represents the angle of the knee joint. Letters beside the curves inform on the data values at knee angles corresponding to configurations

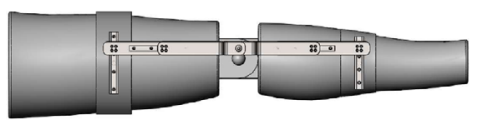

(a)
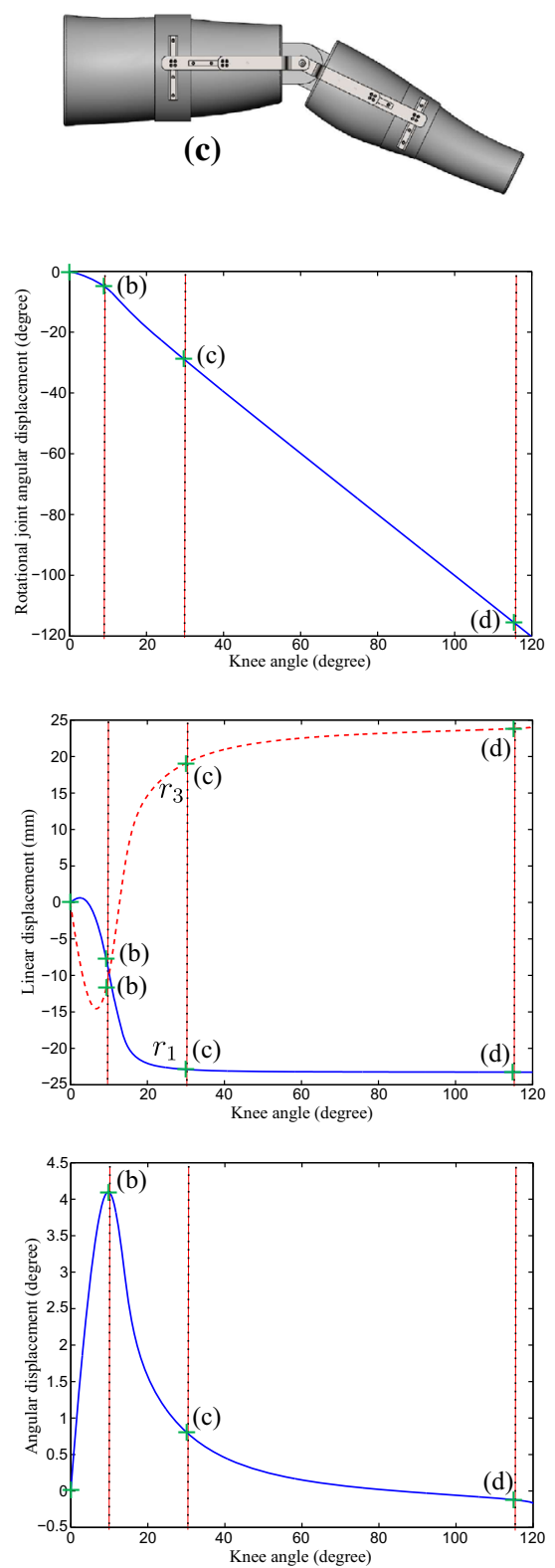

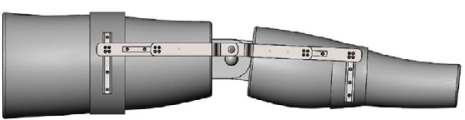

(b)
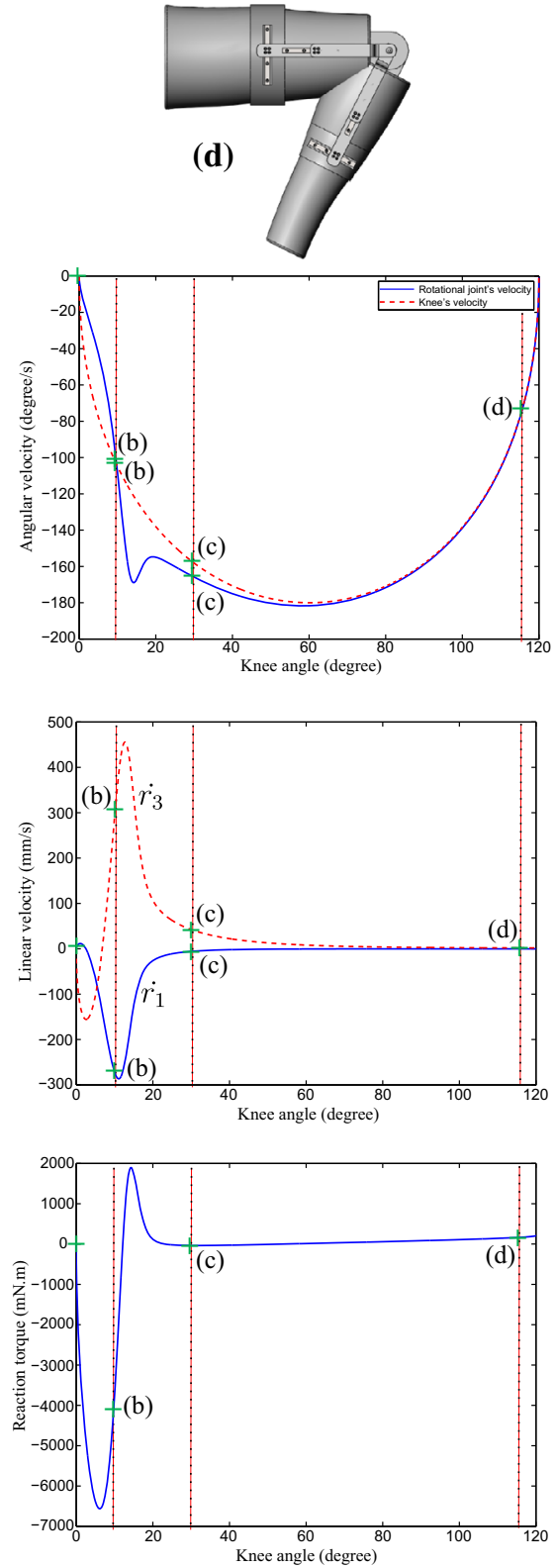

suited for the design of orthoses that automatically align with the anatomical joints they protect. The conditions of the self-adjustment in horizontal and vertical directions are formulated and analyzed. This study is performed for the knee joint, modeled here as a simple pivot joint to simplify the calculations and allow a better understanding of the system behavior during the different operating phases, but the proposed principles could apply to any other joint as well.

One typical PRP mechanism that performs horizontal or vertical self-adjustment motions, leading the orthosis axis of rotation to be aligned with the 
anatomical joint's one, is introduced. The vertical adjustment is performed after the horizontal one. The mechanisms must be attached to the limb in their singular configuration. When they get out of this configuration, the restoring torque produced at the attachment moves the mechanism toward its functioning position, the orthosis axis being in the symmetry plane of the leg after horizontal auto-adjustment and aligned with the knee after vertical auto-adjustment. After self-adjustment, the sliding axes can be fixed. The mechanism becomes again a classic orthosis (this applies both for passive and active devices).

It can be noticed that a higher torque in the calf attachment, synonymous with a larger deformation of this component, means a more difficult self-adjustment. On the contrary, the smaller these values, the better the performance of the mechanism. Hence the deformation of the calf attachment as well as the value of the torsional moment it creates before the selfadjustment movement can be used as performance criteria measuring the efficiency of different mechanisms.

While this study is limited to a simple case for the above-mentioned reasons, it would be interesting to study whether the principle of adding additional prismatic joints to design self adjusting mechanisms can be extended to more complex systems. In particular, it would be interesting to study if the pin joint of the proposed designs may be replaced with a polycentric joint with a variable instantaneous center of rotation (see Fig. 1) to better fit complex movements of a real knee joint. One can expect that the advantage of the proposed designs compared to existing orthoses will remain and that enhanced P-PC-P mechanisms will automatically be positioned in an optimal configuration, i.e. the closest to the anatomical joint. It would also be interesting to extend this study to other spatial mechanisms in order to allow an adaptation to more complex joints as e.g. shoulder or wrist, and/or to take into account their compliance, referring for example to [26].

It can also be noticed that, while the mobility of the skin is not explicitly taken into account in this paper, it will not modify the functioning of the system. As a matter of fact, during self-adjustment motion, the mechanism's principle rotational axis will continue to move as close as possible to the instantaneous helical axis (IHA) of the anatomical joint. If the skin movement is taken into account, this instant screw axis of the two body segments will simply include the skin movement effects besides the bone movements. A device with a polycentric joint allowing to follow the complex knee bone and skin movements would however certainly be more adapted than a simple pivot in this case. One simple way to design such a joint is to measure in advance the relative motion of the two corporal segments, including skin movements, using a 6 axes electro-goniometer [9,27]. The polycentric axis of the device can be designed according to these data.

The authors are aware of the fact that these results are only theoretical at the moment. The next step will be to implement the proposed principles on physical mock-ups and test their behavior in real situation.

\section{References}

1. Wu G, Cavanagh PR (1995) ISB recommendations for standardization in the reporting of kinematic data. J Biomech 28(10):1257-1261

2. Goodfellow J, O'Connor J (1978) The mechanics of the knee and prothesis design. J Bone Joint Surg 60:358-369

3. Winsman J, Veldpaus F, Janssen J, Huson A, Struben P (1980) A three-dimensional mathematical model of the knee-joint. J Biomech 13:677-685

4. Sancisi N, Parenti-Castelli V (2011) A sequentially-defined stiffness model of the knee. Mech Mach Theory 46(12):1920-1928

5. Sancisi N, Parenti-Castelli V (2011) A new kinematic model of the passive motion of the knee inclusive of the patella. J Mech Robot 3(4):041003

6. Markolf KL, Kochan A, Amstutz HC (1984) Measurement of knee stiffness and laxity in patients with documented absence of the anterior cruciate ligament. J Bone Joint Surg 66:242-252

7. Roaas A, Andersson GBJ (1982) Normal range of motion of the hip, knee and ankle joints in male subjects, 30-40 years of age. Acta Orthop Scand 53(2):205-208

8. O'Connor J, Goodfellow J (1978) The mechanics of the knee and prothesis design. J Bone Jt Surg

9. Cai VAD, Bru B, Bidaud P, Hayward V, Gosselin F, Pasqui V (2010) Experimental evaluation of a goniometer for the identification of anatomical joint motions. In: Proceedings of the 13th international conference on climbing and walking robots and the support technologies for mobile machines, pp 1255-1262

10. Walker PS, Kurosawa H, Rovick JS, Zimmerman RA (1985) External knee joint design based on normal motion. J Rehabil Res Dev 22:9-22

11. Aaserude GV, Rubin RH (1987) Polycentric Variable Axis Hinge, United States Patent, Pub. Num. 4.699.129

12. Herzberg T, Albrod A, Orthosis Knee-Joint (2001) United States Patent, Pub. Num. 6,309,368 B1

13. Lamb SR, Moore R (1985) Anatomic Fracture Brace For The Knee, United States Patent, Pub. Num. 4,523,585 
14. Lambert G, Orthosis Knee (2006) United States Patent, Pub. Num. US2006/0089581 A1

15. Reynolds R, Weber R, Landsberger S, Orthesis Knee (2006) United States Patent, Patent Num. US 2006/0211967 A1

16. Spring AN, Kofman J, Lemaire ED (2012) Design and evaluation of an orthotic knee-extension assist. IEEE Trans Neural Syst Rehabil Eng 20(5):678-687

17. Shamaei K, Napolitano P, Dollar AM (2014) Design and functional evaluation of a quasi-passive compliant stance control kneeanklefoot orthosis. IEEE Trans Neural Syst Rehabil Eng 22(2):258-268

18. Weinberg B, Nikitczuk J, Patel S, Patritti B, Mavroidis C, Bonato P, Canavan P (2007) Design, control and human testing of an active knee rehabilitation orthotic device. In: IEEE international conference on robotics and automation, pp 4126-4133

19. Cai D, Bidaud P, Hayward V, Gosselin F (2009) Design of self-adjusting orthoses for rehabilitation. In: Proceedings of the 14th IASTED international conference on robotics and applications, Cambridge. MA, USA, pp 215-223

20. Cai VAD, Bidaud P, Hayward V, Gosselin F, Desailly Eric (2011) Self-adjusting, isostatic exoskeleton for the human knee joint. In: Annual international conference of the IEEE engineering in medicine and biology society, pp 612-618

21. Celebi B, Yalcin M, Patoglu V (2013) ASSISTON-KNEE: a self-aligning knee exoskeleton. In: IEEE/RSJ international conference on intelligent robots and systems (IROS), 2013, Japan, pp 996-1002

22. Jarasse N, Morel G (2011) Connecting a human limb to an exoskeleton. IEEE Trans Robot 28(3):697-710

23. Ergin MA, Patoglu V, Self-Adjusting Knee Exoskeleton A, for Robot-Assisted Treatment of Knee Injuries, Proc. IEEE, RSJ Int. Conf. on Intelligent Robots and Systems, September 25-30, 2011. San Francisco. CA, USA, pp 4917-4922

24. Schorsch JF, Keemink AQL, Stienen AHA, van der Helm FCT, Abbink DA (2014) A novel self-aligning mechanism to decouple force and torques for a planar exoskeleton joint. Mech Sci 5:2935

25. Stienen AHA, Hekman EEG, van der Helm FCT, van der Kooij H (2009) Self-aligning exoskeleton axes through decoupling of joint rotations and translations. IEEE Trans Robot 25:628-633

26. Ding X, Dai JS (2010) Compliance analysis of mechanisms with spatial continuous compliance in the context of screw theory and Lie groups. J Mech Eng Sci Proc I MechE Part C 224(11):2493-2504

27. Kinzel GL, Hall AS, Hillberry BM (1972) Measurement of the total motion between two body segments-i. analytical development. J Biomech 5:93-105 